\title{
High-mobility copper-phthalocyanine field- effect transistors with tetratetracontane passivation layer and organic metal contacts
}

Cite as: J. Appl. Phys. 107, 094503 (2010); https://doi.org/10.1063/1.3354086

Submitted: 18 December 2009 . Accepted: 02 February 2010. Published Online: 06 May 2010

Michael Kraus, Stefan Richler, Andreas Opitz, Wolfgang Brütting, Simon Haas, Tatsuo Hasegawa, Alexander Hinderhofer, and Frank Schreiber

\section{ARTICLES YOU MAY BE INTERESTED IN}

Organic field-effect transistors with high mobility based on copper phthalocyanine Applied Physics Letters 69, 3066 (1996); https://doi.org/10.1063/1.116841

Trap elimination and injection switching at organic field effect transistor by inserting an alkane $\left(\mathrm{C}_{44} \mathrm{H}_{90}\right)$ layer

Applied Physics Letters 90, 033504 (2007); https://doi.org/10.1063/1.2431713

Very high-mobility organic single-crystal transistors with in-crystal conduction channels Applied Physics Letters 90, 102120 (2007); https://doi.org/10.1063/1.2711393

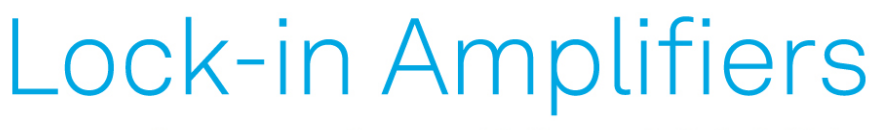
... and more, from DC to $600 \mathrm{MHz}$

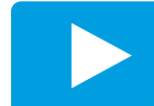

Watch 


\title{
High-mobility copper-phthalocyanine field-effect transistors with tetratetracontane passivation layer and organic metal contacts
}

\author{
Michael Kraus, ${ }^{1, a)}$ Stefan Richler, ${ }^{1}$ Andreas Opitz, ${ }^{1}$ Wolfgang Brütting, ${ }^{1, b)}$ Simon Haas, ${ }^{2}$ \\ Tatsuo Hasegawa, ${ }^{2}$ Alexander Hinderhofer, ${ }^{3}$ and Frank Schreiber ${ }^{3}$ \\ ${ }^{1}$ Institute of Physics, University of Augsburg, 86135 Augsburg, Germany \\ ${ }^{2}$ Photonics Research Institute, National Institute for Advanced Industrial Science and Technology (AIST), \\ Tsukuba 305-8562, Japan \\ ${ }^{3}$ Institute of Applied Physics, University of Tübingen, 72076 Tübingen, Germany
}

(Received 18 December 2009; accepted 2 February 2010; published online 6 May 2010)

\begin{abstract}
We investigate ambipolar charge transport in organic field-effect transistors (OFETs) with copper-phthalocyanine $(\mathrm{CuPc})$ as active material. It is shown that charge carrier mobilities can be increased by at least one order of magnitude using the long-chain alkane tetratetracontane (TTC) as a passivation layer on top of silicon dioxide. TTC and $\mathrm{CuPc}$ films are characterized by atomic force microscopy and x-ray diffraction. TTC forms a highly crystalline layer that passivates electron traps on the $\mathrm{SiO}_{2}$ surface very efficiently and serves as a template for the growth of $\mathrm{CuPc}$ films with significantly improved crystallinity. High electron mobilities comparable to the values reported on single crystals are reached. We show that the contact resistance for hole transport as determined by the transmission line method can be reduced considerably by using organic charge-transfer complexes as top contacts in OFETs based on CuPc. (C) 2010 American Institute of Physics. [doi:10.1063/1.3354086]
\end{abstract}

\section{INTRODUCTION}

Organic semiconductors have been under intensive investigation for the last 30 years. They are believed to be promising candidates for use in electronic and optoelectronic devices. Proposed fields of application are low-cost electronic devices like radio-frequency identification tags or flexible displays. ${ }^{1}$ An interesting feature of organic semiconductors is to exhibit ambipolar charge carrier transport, i.e., transport of both holes and electrons in one single material provided that injection barriers are low and traps at interfaces can be avoided. Ambipolar conduction can give information about the intrinsic charge carrier mobility in organic semiconductors in general and thus is important for a fundamental understanding of transport processes. There are also technical applications for ambipolar transport such as complementary electronic devices or light-emitting fieldeffect transistors (FETs)., ${ }^{2,3}$

It has been demonstrated by time-of-flight measurements on highly pure organic semiconductor crystals in the 1970s and 1980s that ambipolar charge transport in organic semiconductors is possible and that there is no fundamental difference between the transport of electrons and holes. ${ }^{4} \mathrm{Nev}$ ertheless, for a long time, organic semiconductors have been classified as either $p$-type or $n$-type. It has recently become clear that in organic FETs (OFETs) the injecting electrodes or the semiconductor/insulator interface can inhibit the transport of either of the two charge carrier types. ${ }^{5,6}$ In principle there are two ways to achieve ambipolar charge carrier transport and to control the type of charge carrier transported in the active channel. One possibility is the fabrication of

\footnotetext{
${ }^{a)}$ Electronic mail: michael.kraus@physik.uni-augsburg.de.

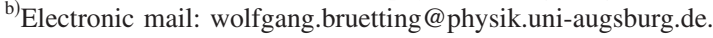

blends of $p$-type and $n$-type materials, where the charge carrier mobilities can be adjusted by the mixing-ratio. ${ }^{7,8}$ The other is treating the substrate with adequate passivation layers. It has been shown that silicon dioxide, which is used very often as gate insulator due to its excellent insulating properties, provides electron traps at the semiconductorinsulator interface and thus hinders electron transport. ${ }^{5}$ Consequently it is important to provide a trap-free interface by adding a passivation layer. With this technique ambipolar transport could be realized in many typically $p$-type materials like pentacene or copper-phthalocyanine $(\mathrm{CuPc}){ }^{6,9}$

$\mathrm{CuPc}$ [Fig. 1(b)] is an organic semiconductor commonly used for OFETs and organic photovoltaic cells but also as a dye for plastics. It is nontoxic and in principle air-stable. Recently, we have demonstrated ambipolar charge carrier transport in evaporated layers of $\mathrm{CuPc}$ on a polymethylmethacrylate (PMMA) passivated substrate. ${ }^{10}$ The type of charge carrier could be controlled by varying the electrode metal. Unipolar electron transport has been observed for $\mathrm{Ca}$ and $\mathrm{Al}$ electrodes, ambipolar transport for $\mathrm{Ag}$ and $\mathrm{Au}$ electrodes and unipolar hole transport for $\mathrm{F}_{4} \mathrm{TCNQ} / \mathrm{Au}$ electrodes.

Here, we use tetratetracontane (TTC) as an alternative passivation layer. TTC is a long-chain alkane molecule
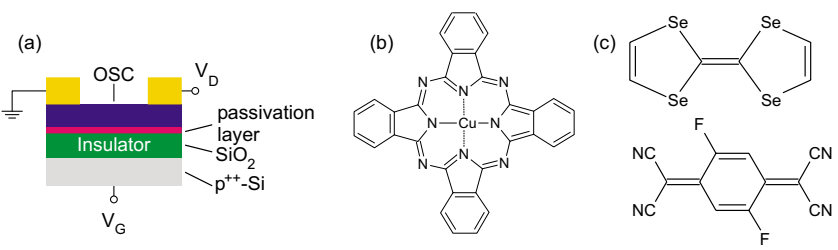

FIG. 1. (Color online) (a) Schematic layout of top-contact OFETs, (b) chemical structure of $\mathrm{CuPc}$, and (c) chemical structure of TSF (upper part) and $\mathrm{F}_{2} \mathrm{TCNQ}$ (lower part). 
$\left(\mathrm{C}_{44} \mathrm{H}_{90}\right)$ that can provide a trap-free surface. ${ }^{11}$ Morphology, flatness, and surface energy of the passivation layer are, among other things, determining factors for the charge carrier transport in OFETs. A highly ordered passivation layer can increase the crystallinity of the semiconductor layer and thus improve charge carrier transport. ${ }^{12}$ We show that crystallinity of CuPc on crystalline TTC is significantly higher than on polymeric PMMA.

For practical applications of OFETs it is important to realize high charge carrier mobilities and low contact resistances. A low contact resistance is important to achieve high currents at a given voltage resulting in low losses. Thus, our goal was to increase charge carrier mobilities by optimizing the passivation layer and to understand and reduce the effect of contact resistance in the devices.

There are several factors contributing to the contact resistance. In most cases the electrode-semiconductor interface is not ideally Ohmic but forms a Schottky contact. This Schottky contact causes an injection barrier depending on the relative positions of the highest occupied molecular orbital (HOMO) and the lowest unoccupied molecular orbital (LUMO) of the semiconductor and the work function of the electrode metal. ${ }^{13}$ Additionally, high temperature during deposition of electrodes onto organic films may lead to diffusion of metal atoms into the organic semiconductor layer or cause damages in the uppermost organic layers. ${ }^{14-16}$

To analyze these effects we used organic charge-transfer (CT) complexes as electrode materials. ${ }^{17-19}$ These materials are combinations of the organic donor materials tetraselenafulvalene (TSF) and tetrathiafulvalene (TTF), respectively, with the organic acceptor material tetracyanoquinodimethane (TCNQ) and its fluorinated derivatives. OFETs with top contacts of these compounds are compared to OFETs with conventional gold contacts.

\section{EXPERIMENTAL}

Transistors were fabricated on highly doped $p^{++}-\mathrm{Si}$ wafers with $320 \mathrm{~nm}$ thermally grown $\mathrm{SiO}_{2}$ acting as gate electrode and gate dielectric, respectively. A sketch of the OFETs can be seen in Fig. 1(a). The wafers have been purchased from Si-Mat, Germany. Cleaning of the wafers was performed in an ultrasonic bath with acetone and isopropanol successively. After cleaning, the $\mathrm{SiO}_{2}$ surface was treated with different passivation layers: TTC and PMMA.

TTC has been purchased from Sigma Aldrich and has been used without any further purification. Approximately 20 $\mathrm{nm}$ of TTC are thermally evaporated at a pressure of 1.0 $\times 10^{-6}$ mbar and a rate of $0.15 \AA / s$. PMMA was spin-coated from a 1.0 wt $\%$ solution in toluene. CuPc has been purchased from Sigma Aldrich as sublimation grade and additionally purified by temperature gradient sublimation. $\mathrm{CuPc}$ was evaporated in a different vacuum chamber at a pressure of $1.0 \times 10^{-7} \mathrm{mbar}$ and a rate of $0.2 \AA / \mathrm{s}$. In our OFETs we used a $25 \mathrm{~nm}$ thick layer of CuPc deposited on top of the passivated substrates.

The surface morphology of all layers (PMMA, TTC, and $\mathrm{CuPc}$ ) was characterized by atomic force microscopy (AFM) and $\mathrm{x}$-ray diffraction (XRD). AFM measurements were per- formed in Augsburg using a Thermo Microscopes Autoprobe CP-Research. XRD measurements were performed in Tübingen using a GE/Seifert x-ray diffractometer using $\mathrm{Cu} K_{\alpha 1}$ line $(\lambda=1.54 \AA)$.

For the transistors, top source and drain contacts were evaporated through a shadow mask with various channel lengths in the range of 30-350 $\mu \mathrm{m}$. In Augsburg, we fabricated samples with gold and silver electrodes and a channel width of $10 \mathrm{~mm}$. In Tsukuba, we also prepared samples (channel width $800 \mu \mathrm{m}$ ) with gold contacts and additionally samples with top contacts of "organic metals," namely the CT complexes TSF-F ${ }_{2}$ TCNQ, TTF-TCNQ, TTF-FTCNQ, TTF-F 2 TCNQ by thermal evaporation of the CT compounds. ${ }^{19}$ The thickness of the contacts was $50 \mathrm{~nm}$ in the case of gold and silver and $200 \mathrm{~nm}$ in the case of the organic metals. The chemical structure of $\mathrm{TSF}-\mathrm{F}_{2} \mathrm{TCNQ}$, which showed the best results, can be seen in Fig. 1(c). Transistor characteristics were measured using a Keithley 4200 Semiconductor Parameter Analyzer in Augsburg and an Agilent E5270A in Tsukuba.

Charge carrier mobility and contact resistance were determined using the so-called transmission line method (TLM). ${ }^{20}$ This technique allows to extract the charge carrier mobility $\mu_{\mathrm{TLM}}$ without the effect of a contact resistance. To do so, the total resistance of the OFET $R_{\text {total }}$ is split into the contact resistance $R_{\mathrm{C}}$ and the channel resistance which is proportional to the channel length $L$. The total resistance is then given by

$$
R_{\text {total }}=R_{\mathrm{C}}+\frac{L}{\mu_{\mathrm{TLM}} W C_{\mathrm{i}}\left(V_{\mathrm{G}}-V_{\mathrm{T}}\right)},
$$

with the channel width $W$, the specific insulator capacitance $C_{\mathrm{i}}$, the gate voltage $V_{\mathrm{G}}$ and the threshold voltage $V_{\mathrm{T}}$. Thus, a plot of the total resistance as a function of the channel length allows to determine $\mu_{\mathrm{TLM}}$ (slope of the linear fit) and $R_{\mathrm{C}}$ (offset of the linear fit). For better comparability to data given in literature the product of the contact resistance and the channel width $R_{\mathrm{C}} \times W$ is used.

\section{RESULTS AND DISCUSSION}

\section{A. Morphology}

The morphology of the interface between the insulator and the organic semiconductor is crucial for the device performance because the transport channel is located in the undermost layers of the semiconductor. Figure 2(a) shows an AFM image of a TTC covered thermally oxidized Si wafer. The thickness of the TTC layer is approximately $20 \mathrm{~nm}$. It can be seen in the height profile along the line [Fig. 2(b)] that TTC forms very smooth terraces of constant height. The height difference between the terraces is always approximately $5 \mathrm{~nm}$. This corresponds very well to the length of the molecule leading to the conclusion that TTC molecules are standing upright on the $\mathrm{SiO}_{2}$ surface. ${ }^{21,22}$ An AFM image of PMMA (not shown here) shows a very smooth surface without any substructures and an rms roughness of approximately $0.25 \mathrm{~nm}$.

Figure 3 shows X-ray reflectivity data of a TTC covered $\mathrm{SiO}_{2}$ substrate. A series of seven Bragg reflections are ob- 
(a)
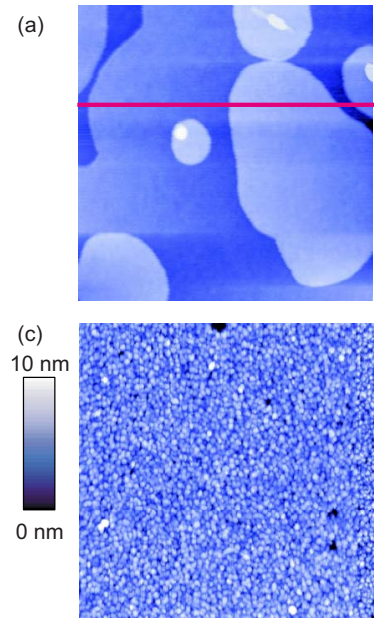

(b)
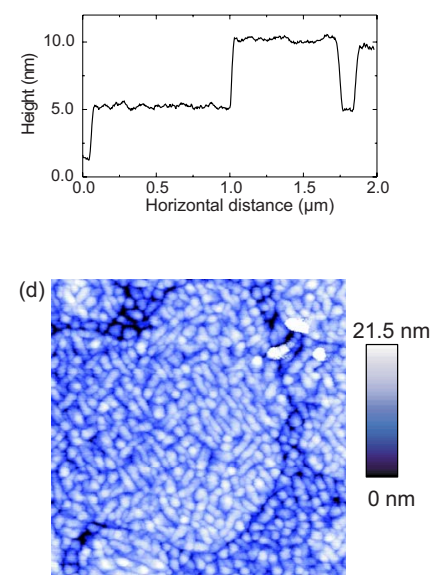

FIG. 2. (Color online) (a) AFM image of TTC on $\mathrm{SiO}_{2}$, (b) height profile along the line in (a), (c) AFM image of CuPc on PMMA and (d) $\mathrm{CuPc}$ on TTC, $\left(\right.$ size $2 \times 2 \mu \mathrm{m}^{2}$ ).

servable indicating a highly crystalline TTC layer. A fit with the Parrat-formalism allows for the extraction of the electron density profile in the direction normal to the surface ${ }^{23}$ (see inset of Fig. 3). From the electron density profile, a lattice spacing of $5.80 \mathrm{~nm}( \pm 0.01 \mathrm{~nm})$ for the TTC film can be derived. In addition it can be directly seen in the electron density profile that the first two layers of TTC are completely filled, the third to fifth layer are successively less filled. These findings from $x$-ray scattering correspond very well to the AFM data presented in Figs. 2(a) and 2(b).

The full width at half-maximum (FWHM) of the rocking scan on the first Bragg reflection indicates that the mosaicity of the TTC layer is very small $\left(\mathrm{FWHM}<0.03^{\circ}\right)$. We conclude that TTC forms a very well defined crystalline film where the individual molecules are standing upright and thus form very smooth terraces.

As a next step we deposited $25 \mathrm{~nm}$ of $\mathrm{CuPc}$ on substrates covered with PMMA or TTC to analyze the different growth mechanisms of $\mathrm{CuPc}$ on these types of passivation layers. Figure 2 shows AFM images of $\mathrm{CuPc}$ on a PMMA treated substrate (c) and $\mathrm{CuPc}$ on a TTC treated substrate (d). The polycrystallinity of the CuPc layer can clearly be seen. While on PMMA CuPc forms randomly oriented crystallites with an average diameter of approximately $50 \mathrm{~nm}$, in the case of TTC as substrate the crystallites are elongated with an aver-

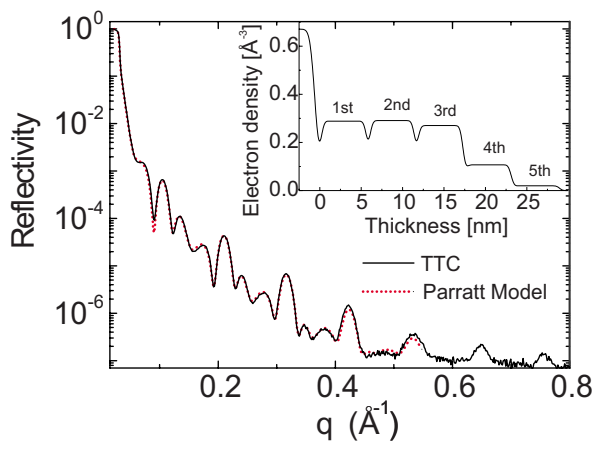

FIG. 3. (Color online) X-ray reflectivity and fit of a TTC covered $\mathrm{SiO}_{2}$ substrate. The inset shows the fitted electron density profile.

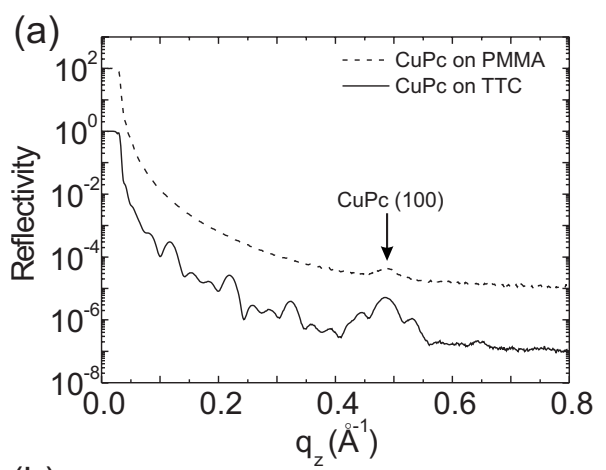

(b)

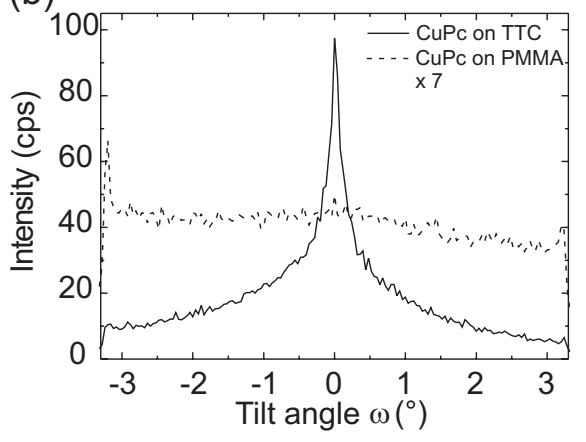

FIG. 4. (a) X-ray reflectivity of $25 \mathrm{~nm} \mathrm{CuPc}$ on top of TTC (solid line) and PMMA (dashed line). (b) Rocking curves of the Bragg reflection at $q_{z}$ $=0.487 \AA^{-1}$. For better visibility the dashed curves have been multiplied by a constant factor.

age size of approximately $250 \times 80 \mathrm{~nm}^{2}$. Additionally, they are preferentially oriented along certain directions. The terraces formed by the TTC layer are still visible indicating that individual crystallites do not grow over a terrace step. However, the height of the crystallites is much larger than the TTC terrace step height so that they are in contact to each other which is crucial for charge carrier transport.

Figure 4(a) depicts x-ray reflectivity data of $25 \mathrm{~nm} \mathrm{CuPc}$ on top of TTC (solid line) and PMMA (dashed line). There is a clear peak at $q_{z}=0.487 \AA^{-1}$ corresponding to the (100)peak of the $\alpha$-phase of $\mathrm{CuPc}^{24,25}$ In this phase, CuPc molecules are standing nearly upright on the substrate and are closely packed in the direction parallel to the substrate with a horizontal lattice spacing of approximately $12.9 \AA$ between two $\mathrm{Cu}$ atoms. Such close packing in horizontal direction is favorable for charge carrier transport in OFETs. The intensity of the Bragg peak of CuPc on TTC is more than one order of magnitude higher than on PMMA. This means that the alignment of the $\mathrm{CuPc}$ crystallites perpendicular to the substrate is better on TTC. The rocking curves shown in Fig. 4(b) support this assumption. The FWHM of the CuPc (100) peak on TTC is approximately $0.26^{\circ}$ whereas on PMMA it is larger than $2^{\circ}$.

From the AFM and XRD data, it can be concluded that $\mathrm{CuPc}$ forms larger crystallites on TTC than on PMMA, which show (in some degree) preferential orientation. This improvement can be understood in terms of a kind of pseudoepitaxy as the result of anisotropic surface diffusion during the growth of CuPc. However, other effects like the surface energy can play a role, too. The latter is linked to the water contact angle. It has been shown that charge carrier mobility increases with increasing water contact angle $\alpha$ of the passi- 

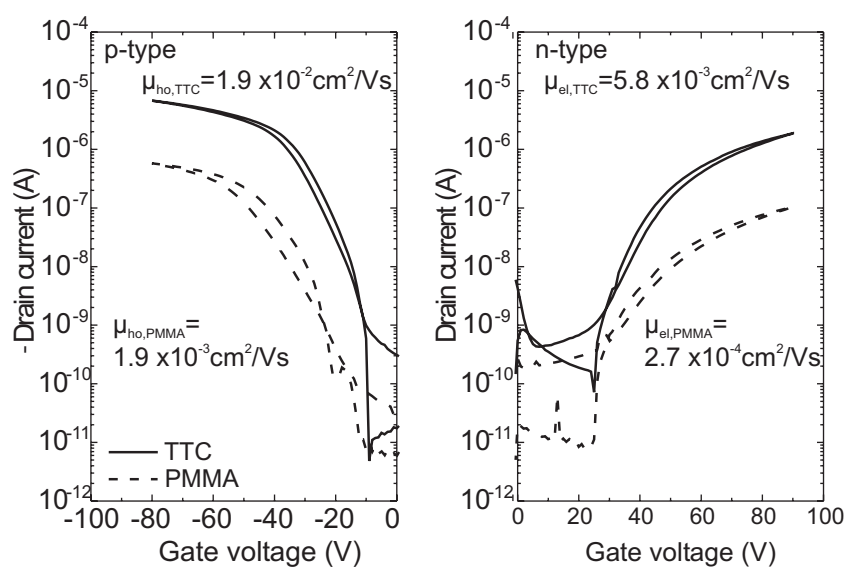

FIG. 5. Transfer characteristics and charge carrier mobilities of OFETs with TTC and PMMA passivation layers (channel length $L=120 \mu \mathrm{m}$, channel width $W=1 \mathrm{~cm}, V_{\mathrm{D}}= \pm 10 \mathrm{~V}$, and Ag electrodes).

vation layer. $^{26-28}$ We found $\alpha_{\mathrm{TTC}} \approx 105^{\circ}$ and $\alpha_{\mathrm{PMMA}} \approx 85^{\circ}$ which proves that TTC is more hydrophobic than PMMA leading to larger crystal size of CuPc. A more hydrophobic surface can lead to a better crystallinity of the semiconductor. $^{26}$

In conclusion it can be stated that TTC is more favorable as passivation layer than PMMA because of its well ordered growth and the lower surface energy which together lead to a significantly increased crystallinity of the CuPc active layer.

\section{B. Electrical measurements}

The differences in morphology are well reflected in the OFET characteristics. Transfer characteristics of OFETs with TTC (solid lines) and PMMA (dashed lines) passivation layers and Ag electrodes are shown in Fig. 5. Both types of samples are ambipolar. The left hand side depicts the hole transport regime (negative gate voltage) at a drain voltage of $V_{\mathrm{D}}=-10 \mathrm{~V}$ whereas the right hand side shows the electron transport regime (positive gate voltage) at a given drain voltage of $V_{\mathrm{D}}=+10 \mathrm{~V}$. While the threshold voltage is almost identical for PMMA and TTC treated wafers $\left(V_{\mathrm{T}} \approx-36 \mathrm{~V}\right.$ for holes and $V_{\mathrm{T}} \approx+50 \mathrm{~V}$ for electrons), the drain current is significantly higher for TTC-based samples. Transistors on TTC reveal a considerably increased charge carrier mobility (see Fig. 5): by a factor of 10 for hole transport and a factor of 20 for electron transport. Electron mobility in our devices reaches up to $5.8 \times 10^{-3} \mathrm{~cm}^{2} / \mathrm{V}$ s which is best for $\mathrm{CuPc}$ thin-films and almost equal to values reported on $\mathrm{CuPc}$ single crystal OFETs. ${ }^{29}$ Another important aspect is the asymmetry between hole and electron transport. On PMMA the respective mobilities differ by a factor of 7 , whereas on TTC they only differ by a factor of 2 , which shows that charge carrier mobilities are affected seriously by the semiconductor-insulator interface. This effect is different for electrons and holes indicating that the intrinsic transport properties of $\mathrm{CuPc}$ may be similar for both charge carrier types but electrons and holes are affected by the passivation layer in a different way as already mentioned in literature. ${ }^{4}$ Actually, OFETs with Au top contacts revealed comparable transfer characteristics and charge carrier mobilities as will be discussed below.
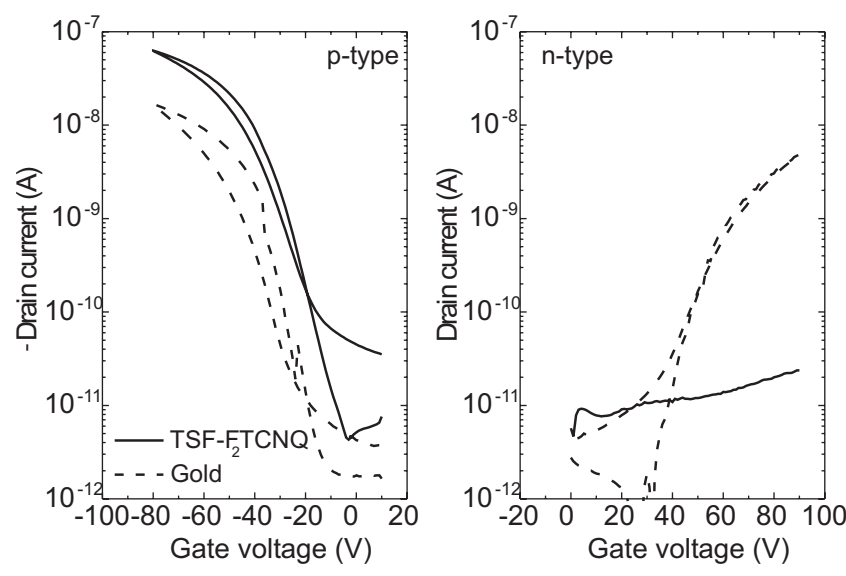

FIG. 6. Transfer characteristics of CuPc-OFETs on TTC with TSF-F 2 TCNQ and gold electrodes (channel length $L=100 \mu \mathrm{m}$, channel width $W$ $=800 \mu \mathrm{m}), V_{\mathrm{D}}= \pm 2 \mathrm{~V}$.

The difference between PMMA and TTC underlines the importance of increasing crystal grain size in order to reach higher mobilities. Due to the larger grain size in OFETs based on TTC there are less grain boundaries in a charge carrier path which hinder the transport.

Another factor influencing charge carrier mobility is the dielectric constant of the passivation layer. In our case, we determined $\varepsilon_{\mathrm{TTC}} \approx 2.5$ and $\varepsilon_{\mathrm{PMMA}} \approx 3.6$ from capacitance measurements. It has been demonstrated that charge carrier mobility in OFETs decreases with increasing dielectric constant of the insulator because of the formation of Fröhlich polarons. $^{30}$

\section{Reduction of contact resistance}

For ambipolar OFETs with one single electrode material there is always an energetic misalignment concerning the injection for at least one charge carrier type. This misalignment increases the contact resistance due to the formation of a Schottky barrier.

It has been shown that organic metals can improve charge carrier injection and lower the contact resistance in comparison to conventional metal electrodes in the case of OFETs with dibenzoTTF (DBTTF)-TCNQ or neat DBTTF active layers, which are chemically very similar to the materials used as organic metal electrodes mentioned above, and also in the case of dinaphtho-thieno-thiophene. ${ }^{18,31,32}$ These improvements also work in the case of $\mathrm{CuPc}$, which is chemically and morphologically totally different from the organic metals.

We fabricated OFETs with top-contacts of gold and various organic metals (TSF-F 2 TCNQ, TTF-TCNQ, TTFFTCNQ, and TTF-F 2 TCNQ) with channel lengths down to $30 \mu \mathrm{m}$. Such small channel lengths allow a more precise evaluation of the contact resistance by TLM. The device with Au contacts revealed ambipolar charge carrier transport like the samples shown above. All devices with organic metals showed unipolar hole transport but only in the case of TSF-F $F_{2}$ TCNQ mobilities were comparable to OFETs with Au contacts. Figure 6 depicts transfer characteristics of a sample with $\mathrm{Au}$ and a sample with TSF-F 2 TCNQ top contacts. 


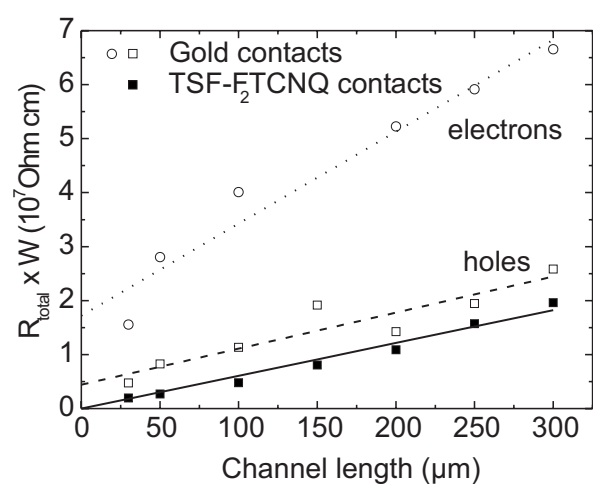

FIG. 7. Total resistance of the hole and electron transport regimes for $\mathrm{CuPc}$ OFETS on TTC with gold (open symbols) and TSF-F 2 TCNQ (closed symbols) contacts and linear fits of the data. The contact resistance is determined from the offset of the linear fit.

Samples with TSF- $F_{2}$ TCNQ electrodes show significantly higher currents in the hole transport regime as compared to samples with gold contacts but do not show any electron transport. To understand this effect it is important to look at the energy levels of the materials used here. The ionization potential of $\mathrm{CuPc}$ is $5.0 \mathrm{eV}$ (HOMO), the electron affinity is $3.2 \mathrm{eV}$ (LUMO). ${ }^{33,34}$ This indicates that the HOMO energy level should fit very well to the Fermi level of gold [work function $\Phi_{\mathrm{Au}} \approx 5.0 \mathrm{eV}$, determined by Kelvin probe measurements as bottom contact in ultrahigh vacuum (UHV)].

There is almost no data available on the work function of organic metals. Only the work function of TTF-TCNQ was estimated to be in between 4.6 and $4.8 \mathrm{eV}$, slightly lower than $\mathrm{Au}^{35}$ However, TTF-TCNQ was reported to have the lowest work function of all organic metals used here and TSF- $F_{2}$ TCNQ the highest. ${ }^{19}$ Since the total difference is very small, it can be assumed that the work function of TSF- $\mathrm{F}_{2} \mathrm{TCNQ}$ is around $5.0 \mathrm{eV}$ and thus comparable to that of $\mathrm{Au}$ in UHV.

Figure 7 compares the total resistance of CuPc-OFETs with gold and TSF- $\mathrm{F}_{2}$ TCNQ contacts as a function of the channel length. Following the TLM method explained in Sec. II, hole mobility and contact resistance can be determined by linear fits of the data. It can be seen from the slope of the fit lines that gold and TSF-F $\mathrm{F}_{2} \mathrm{TCNQ}$ show equal hole mobilities as summarized in Table I.

Contact resistances for hole transport determined by TLM exhibit an interesting feature: a finite resistance $\left(R_{\mathrm{C}, \mathrm{Au}} \times W \approx 4.4 \times 10^{6} \Omega \mathrm{cm}\right)$ for gold electrodes is measured whereas the contact resistance for TSF-F $F_{2}$ TCNQ electrodes is too small to be determined by TLM and almost negligible in comparison to Au contacts. This shows that

TABLE I. Charge carrier mobility and contact resistance of CuPc-OFETs on TTC covered $\mathrm{SiO}_{2}$ substrates with Au and TSF- $\mathrm{F}_{2}$ TCNQ contacts as determined by TLM.

\begin{tabular}{lcccc}
\hline \hline Electrode material & $\begin{array}{c}\mu_{\mathrm{ho}} \\
\left(\mathrm{cm}^{2} / \mathrm{V} \mathrm{s}\right)\end{array}$ & $\begin{array}{c}\mu_{\mathrm{el}} \\
\left(\mathrm{cm}^{2} / \mathrm{V} \mathrm{s}\right)\end{array}$ & $\begin{array}{c}R_{\mathrm{C}, \mathrm{ho}} \times W \\
(\Omega \mathrm{cm})\end{array}$ & $\begin{array}{c}R_{\mathrm{C}, \mathrm{el}} \times W \\
(\Omega \mathrm{cm})\end{array}$ \\
\hline Au & $7.3 \times 10^{-3}$ & $2.8 \times 10^{-3}$ & $4.4 \times 10^{6}$ & $1.7 \times 10^{7}$ \\
TSF-F 2 TCNQ & $7.4 \times 10^{-3}$ & - & $<1.0 \times 10^{6}$ & - \\
\hline \hline
\end{tabular}

hole injection is improved significantly by using TSF- $F_{2}$ TCNQ electrodes. The reduction of the contact resistance can be attributed to the good energy level alignment of $\mathrm{CuPc}$ HOMO and TSF-F 2 TCNQ work function which reduces the injection barrier height for holes and eliminates electron injection. Such misalignment effect is one of the main influences on the contact resistance.

It is remarkable that samples with Au electrodes show ambipolar charge carrier transport although the work function of $\mathrm{Au}$ (in UHV) fits very well to the HOMO of $\mathrm{CuPc}$. However, the work function of the metal and the HOMO level cannot be directly compared because of the formation of interface dipoles at the metal-organic interface that break the vacuum level alignment. It has been reported that the Fermi level of gold is about $0.7 \mathrm{eV}$ above the HOMO level of $\mathrm{CuPc}$ in the case of CuPc deposited on gold. ${ }^{36}$ In the case of gold top contacts the situation is more complicated due to the possible diffusion of gold atoms into the semiconductor. Nevertheless, a similar energy level alignment has been observed. ${ }^{37}$ In contrast, TSF- $F_{2}$ TCNQ contacts yield only hole transport despite a work function comparable to that of $\mathrm{Au}$. The key point is that we have an organic-organic interface in this case. In contrast to the metal-organic interface discussed above, these kinds of interfaces are usually dominated by vacuum level alignment. ${ }^{38}$ Due to this vacuum level alignment the Fermi level of TSF- $\mathrm{F}_{2} \mathrm{TCNQ}$ remains relatively unchanged when evaporated on CuPc. Another point is the deposition of metal on molecular organic semiconductors which does not lead to the formation of a sharp metalsemiconductor interface but to the diffusion of metal atoms into the semiconductor. ${ }^{15,16,39}$ Furthermore, the high temperature during metal evaporation can damage the uppermost CuPc layer and form defect states. ${ }^{40}$ These effects are supposed to lower the effective work function of Au top contacts in comparison to neat $\mathrm{Au}$ films. It has been reported that sputtered indium tin oxide can induce defects in the organic semiconductor layers of organic light-emitting diodes allowing for electron injection. ${ }^{14}$ Since evaporation temperatures of organic metals are only around $170{ }^{\circ} \mathrm{C}$ damage does not occur in that case. Additionally, the molecular size of the organic metals is significantly larger which hinders a diffusion into the CuPc layer. Consequently, the effective work function of the organic metal top contacts is not shifted and thus larger than that of Au contacts which promotes hole injection. This finding shows that although electron injection becomes possible with Au top contacts the shift in the work function is in a range so that hole injection still remains favorable.

\section{SUMMARY}

In conclusion, we have compared TTC and PMMA as surface passivation layers in ambipolar OFETs with CuPc as active layer and $\mathrm{Au}$ or $\mathrm{Ag}$ as top electrodes. We have shown that TTC forms highly crystalline films with molecules standing upright on $\mathrm{SiO}_{2}$ surfaces and forming very smooth terraces. The morphology of the CuPc layer depends strongly on the surface properties of the underlying substrate. The size of $\mathrm{CuPc}$ crystallites could be increased significantly 
when changing from PMMA to TTC as passivation layer. Additionally, the crystallites show a preferential orientation on TTC. Due to the higher crystallinity charge carrier mobilities could be raised by at least one order of magnitude for holes and electrons ( $\mu_{\text {ho,max }}=1.9 \times 10^{-2} \mathrm{~cm}^{2} / \mathrm{V} \mathrm{s}$ and $\mu_{\mathrm{el}, \max }=5.8 \times 10^{-3} \mathrm{~cm}^{2} / \mathrm{V} \mathrm{s}$ ).

After optimizing the surface passivation layer we focused on the reduction in the contact resistance. OFETs with top contacts made of the organic CT complex TSF- $\mathrm{F}_{2} \mathrm{TCNQ}$ have been compared to those with contacts of ordinary metals. OFETs with gold contacts revealed ambipolar transport whereas only hole transport could be observed with TSF- $F_{2}$ TCNQ top contacts, although nominal work functions should be comparable. However, the relevant quantity is the interface energy level alignment, which is different for the two systems. An additional effect can be the diffusion of $\mathrm{Au}$ atoms into the $\mathrm{CuPc}$ layer due to the high evaporation temperatures of gold. These effects are supposed to enable electron injection from $\mathrm{Au}$ contacts due to a change in the gold Fermi level. Contact resistance for hole injection has been reduced significantly using TSF- $\mathrm{F}_{2}$ TCNQ which proves the good alignment of the CuPc HOMO level and the TSF-F $F_{2}$ TCNQ work function.

\section{ACKNOWLEDGMENTS}

We would like to thank Yutaka Noguchi and Hisao Ishii from the University of Chiba, Japan, for fruitful discussions about TTC, Toshikazu Yamada (AIST) for technical support, and Jens Pflaum from the Universities of Stuttgart and Würzburg for the purification of CuPc. We thank the Deutsche Forschungsgemeinschaft (DFG) for their financial support through SFB 484 at Augsburg and through Project No. Schr700/13-1 at Tübingen as well as the Japan Society for the Promotion of Science (JSPS) for financing visits to Tsukuba.

${ }^{1}$ H. Sirringhaus, Adv. Mater. 17, 2411 (2005).

${ }^{2}$ A. Babel, J. D. Wind, and S. A. Jenehke, Adv. Funct. Mater. 14, 891 (2004).

${ }^{3}$ J. Zaumseil, R. H. Friend, and H. Sirringhaus, Nature Mater. 5, 69 (2006)

${ }^{4}$ N. Karl, in Organic Semiconductors, Landolt-Boernstein (New Series) Chap. Organic Semiconductors, Group III, Vol. 41E, edited by O. Madelung, M. Schulz, and H. Weiss (Springer, Berlin, 2000).

${ }^{5}$ L. L. Chua, J. Zaumseil, J. F. Chang, E. C. W. Ou, P. K. H. Ho, H Sirringhaus, and R. H. Friend, Nature (London) 434, 194 (2005).

${ }^{6}$ T. Yasuda, T. Goto, K. Fujita, and T. Tsutsui, Appl. Phys. Lett. 85, 2098 (2004).

${ }^{7}$ E. J. Meijer, D. M. De Leeuw, S. Setayesh, E. Van Veenendaal, B. H. Huisman, P. W. M. Blom, J. C. Hummelen, U. Scherf, and T. M. Klapwijk, Nature Mater. 2, 678 (2003).

${ }^{8}$ A. Opitz, M. Bronner, and W. Brütting, J. Appl. Phys. 101, 063709 (2007).
${ }^{9}$ M. Ahles, R. Schmechel, and H. v. Seggern, Appl. Phys. Lett. 87, 113505 (2005).

${ }^{10}$ A. Opitz, M. Kraus, M. Bronner, J. Wagner, and W. Brütting, New J. Phys. 10, 065006 (2008).

${ }^{11}$ S. Ogawa, Y. Kimura, M. Niwano, and H. Ishii, Appl. Phys. Lett. 90, 033504 (2007).

${ }^{12}$ K. Yamaguchi, S. Takamiya, M. Minami, Y. Doge, Y. Nishide, H. Osuga, K. Uno, and I. Tanaka, Appl. Phys. Lett. 93, 043302 (2008).

${ }^{13}$ X. Cheng, Y.-Y. Noh, J. Wang, M. Tello, J. Frisch, R.-P. Blum, A. Vollmer, J. Rabe, N. Koch, and H. Sirringhaus, Adv. Funct. Mater. 19, 2407 (2009).

${ }^{14}$ G. Parthasarathy, P. E. Burrows, V. Khalfin, V. G. Kozlov, and S. R. Forrest, Appl. Phys. Lett. 72, 2138 (1998).

${ }^{15}$ A. C. Dürr, F. Schreiber, M. Kelsch, H. D. Carstanjen, and H. Dosch, Adv. Mater. 14, 961 (2002).

${ }^{16}$ A. C. Dürr, F. Schreiber, M. Kelsch, H. D. Carstanjen, H. Dosch, and O. H. Seeck, J. Appl. Phys. 93, 5201 (2003).

${ }^{17}$ D. Jérome and H. Schulz, Adv. Phys. 31, 299 (1982).

${ }^{18}$ Y. Takahashi, T. Hasegawa, Y. Tokura, K. Nishimura, and G. Saito, Appl. Phys. Lett. 86, 063504 (2005)

${ }^{19}$ Y. Takahashi, T. Hasegawa, Y. Abe, Y. Tokura, and G. Saito, Appl. Phys. Lett. 88, 073504 (2006).

${ }^{20}$ S. Luan and G. W. Neudeck, J. Appl. Phys. 72, 766 (1992).

${ }^{21}$ J.-P. Gorce, S. Spells, X.-B. Zeng, and G. Ungar, J. Phys. Chem. B 108, 3130 (2004).

${ }^{22}$ N. Abiko, K. Sugi, T. Suenaga, Y. Kimura, H. Ishii, and M. Niwano, Jpn. J. Appl. Phys., Part 1 45, 442 (2006).

${ }^{23}$ L. G. Parratt, Phys. Rev. 95, 359 (1954).

${ }^{24}$ O. Berger, W.-J. Fischer, B. Adolphi, S. Tierbach, V. Melev, and J. Schreiber, J. Mater. Sci.: Mater. Electron. 11, 331 (2000).

${ }^{25}$ A. Hoshino, Y. Takenaka, and H. Miyaji, Acta Crystallogr. B 59, 393 (2003).

${ }^{26}$ C. K. Song, B. W. Koo, S. B. Lee, and D. H. Kim, Jpn. J. Appl. Phys., Part 141,2730 (2002).

${ }^{27}$ J. Veres, S. Ogier, G. Lloyd, and D. de Leeuw, Chem. Mater. 16, 4543 (2004).

${ }^{28}$ S. Grecu, M. Roggenbuck, A. Opitz, and W. Brütting, Org. Electron. 7, 276 (2006).

${ }^{29}$ R. W. I. de Boer, A. F. Stassen, M. F. Craciun, C. L. Mulder, A. Molinari, S. Rogge, and A. F. Morpurgo, Appl. Phys. Lett. 86, 262109 (2005).

${ }^{30}$ I. N. Hulea, S. Fratini, H. Xie, C. L. Mulder, N. N. Iossad, G. Rastelli, S. Ciuchi, and A. F. Morpurgo, Nature Mater. 5, 982 (2006).

${ }^{31}$ K. Shibata, K. Ishikawa, H. Takezoe, H. Wada, and T. Mori, Appl. Phys. Lett. 92, 023305 (2008).

${ }^{32}$ S. Haas, Y. Takahashi, K. Takimiya, and T. Hasegawa, Appl. Phys. Lett. 95, 022111 (2009).

${ }^{33}$ O. V. Molodtsova, T. Schwieger, and M. Knupfer, Appl. Surf. Sci. 252, 143 (2005).

${ }^{34}$ S. Krause, M. B. Casu, A. Scholl, and E. Umbach, New J. Phys. 10, 085001 (2008).

${ }^{35}$ K. Shibata, H. Wada, K. Ishikawa, H. Takezoe, and T. Mori, Appl. Phys. Lett. 90, 193509 (2007).

${ }^{36}$ L. Yan, N. J. Watkins, S. Zorba, Y. Gao, and C. W. Tang, Appl. Phys. Lett. 79, 4148 (2001)

${ }^{37}$ L. Lozzi, S. Santucci, and S. L. Rosa, J. Vac. Sci. Technol. A 22, 1477 (2004).

${ }^{38}$ N. Koch, ChemPhysChem 8, 1438 (2007).

${ }^{39}$ M. Scharnberg, J. Hu, J. Kanzow, K. Rätzke, R. Adelung, F. Faupel, C. Pannemann, U. Hilleringmann, S. Meyer, and J. Pflaum, Appl. Phys. Lett. 86, 024104 (2005).

${ }^{40}$ J. G. Xue, B. P. Rand, S. Uchida, and S. R. Forrest, J. Appl. Phys. 98, 124903 (2005) 